\section{O Uso de Carbonato de Litio no Tratamento da Tiveotoxicose Induzida por Amiodarona}

\section{RESUMO}

Dentre as disfunções tireoidianas induzidas por amiodarona, a tireotoxicose é a mais grave e associada a maior morbi-mortalidade. $\bigcirc$ tratamento consiste no emprego de altas doses de drogas antitireoidianas e corticoesteróides, de forma isolada ou combinada. Outras terapias associadas foram propostas para o tratamento dos casos refratários. Relatamos o caso de um paciente de 40 anos com história de miocardiopatia dilatada idiopática que desenvolveu um quadro grave de tireoidite induzida por amiodarona após transplante cardíaco. Este paciente não respondeu à terapia inicial com doses altas de droga antitireoidiana e corticoesteróides, sendo, então, associado o carbonato de lítio em baixas doses e por curto período de tempo, o que resultou na normalização da função tireoidiana. Neste caso, o emprego do carbonato de lítio mostrou-se como uma terapia adjuvante eficaz e segura no controle da tireotoxicose induzida por amiodarona. (Ara Bras Endocrinol Metab 2005;49/6:991-995)

Descritores: Tratamento; Tireotoxicose; Tireoidite; Amiodarona; Carbonato de lítio

\begin{abstract}
Use of Lithium Carbonate for the Treatment of Amiodarone-Induced Thyrotoxicosis.

Among the amiodarone-induced thyroid dysfunctions, thyrotoxicosis is the most troublesome and with the highest rate of morbidity and mortality. Treatment consists in the use of a high dose of anti-thyroid drugs and steroids in an isolated form or in combination. Association of several other drugs have been proposed for the treatment of refractory cases. In this study we report the case of a 40 y.o. patient, with a history of idiopatic dilated miocardiopathy, who developed severe amioradone-induced thyrotoxicosis after heart transplantation. Since the patient did not respond to an initial treatment consisting of a high dose of anti-thyroid drugs combined with steroids, a low dose of lithium carbonate was added for a short period of time, which resulted in normalization of the thyroid function. In this case, the addition of lithium carbonate to the two other drugs resulted in a successful and safety therapy in controlling amiodarone-induced thyrotoxicosis. (Arq Bras Endocrinol Metab 2005;49/6:991-995)
\end{abstract}

Keywords: Treatment; Thyrotoxicosis; Thyroiditis; Amiodarone; Lithium carbonate

\footnotetext{
A Amiodarona, um potente agente antiarrítmico, apresenta alta concentração de iodo em sua composição (1). A presença do iodo na droga é responsável pela elevada incidência de disfunções tireoidianas, associadas ao seu uso crônico $(2,3)$.
}

apresentação de casos

\author{
Serviço de Endocrinologia e \\ Metabologia, Hospital de \\ Clinicas da Universidade \\ Federal do Paraná - SEMPR, \\ Curitiba, $P R$.
}


A tireotoxicose induzida por amiodarona ocorre em até $23 \%$ dos pacientes e é mais prevalente em áreas com carência de iodo (4). Classifica-se por tireotoxicose tipo 1, aquela com síntese excessiva de hormônios tireoidianos, ou tipo 2, na qual um processo destrutivo é responsável pela liberação do hormônio tireoidiano pré-formado. A distinção clínica entre as duas formas de tireotoxicose induzida por amiodarona é difícil, especialmente porque alguns pacientes apresentam uma forma mista da doença.

Em decorrência do elevado tempo de meia-vida da droga no organismo e da necessidade imperativa de tratamento adequado da tireotoxicose, principalmente em pacientes cardiopatas, várias terapias têm sido propostas. As drogas antitireoidianas e os corticoesteróides amplamente utilizados, de forma isolada ou combinada, parecem não apresentar resultados satisfatórios nos casos mais graves $(2,5,6)$.

O carbonato de lítio, utilizado para tratamento de doenças psiquiátricas, interfere na síntese e reduz a secreção dos hormônios tireoidianos $(7,8)$. O emprego do lítio, em altas doses e de forma prolongada, foi sugerido como uma forma eficiente e segura de tratamento da tireotoxicose induzida por amiodarona, especialmente nas formas mais severas de doença (7).

Relatamos o caso de um paciente com história de miocardiopatia dilatada idiopática, que desenvolveu um quadro grave de tireotoxicose induzida por amiodarona após transplante cardíaco. O emprego de carbonato de lítio em doses baixas e por curto período de tempo demonstrou-se como uma possível alternativa terapêutica nos casos de tireotoxicose induzida por amiodarona.

\section{RELATO DO CASO}

J.A.R., 40 anos, masculino, branco, casado, procedente de Curitiba, foi avaliado no Ambulatório de Tireóide do Serviço de Endocrinologia e Metabologia do Hospital de Clínicas da Universidade Federal do Paraná por tireotoxicose. Apresentava diagnóstico prévio de miocardiopatia dilatada idiopática, tendo sido submetido a transplante cardíaco em dezembro de 2003 , em outro serviço. Relatava uso crônico de amiodarona por sete anos, a qual foi suspensa logo após a realização do transplante.

Em janeiro de 2004, recebeu o diagnóstico de tireotoxicose através de exames laboratoriais de rotina $(\mathrm{TSH}<0,05 \mathrm{mUI} / \mathrm{L} ;$ T4livre $=6,32 \mathrm{ng} / \mathrm{dl} ;$ Trab $=$ negativo; anticorpo anti-TPO= negativo; anticorpo anti$\mathrm{TG}=$ negativo), sendo iniciado tratamento com droga antitireoidiana (metimazol) e encaminhado para avaliação endocrinológica. O paciente havia realizado uma cintilografia de tireóide, a qual demonstrou captação de $0,3 \%$ em 24 horas, porém este exame foi executado em vigência do uso de metimazol.

Em sua primeira consulta neste serviço, realizada em maio de 2004, apresentava queixas de emagrecimento, com redução de $30 \mathrm{~kg}$ em 6 meses, hiporexia, insônia, tremor de extremidades e palpitações ocasionais. Estava em uso de metimazol $50 \mathrm{mg} /$ dia há 4 meses, associado a ciclosporina, micofenolato mofetil e diltiazen. Relatava história de diabetes mellitus diagnosticado após o transplante, controlado apenas com dieta. Vinha em acompanhamento cardiológico periódico com evolução favorável.

Ao exame, encontrava-se em bom estado geral, corado, hidratado, normotenso (130 x 70mmHg), com $\mathrm{FC}=83 \mathrm{bpm}$. A tireóide estava difusamente aumentada, com aproximadamente 30 gramas à palpação. As bulhas cardíacas eram rítmicas, normofonéticas, com sopro sistólico $(+/ 4)$ e campos pleuropulmonares livres. Tremor de extremidades moderado, com pele quente e úmida. Ausência de oftalmopatia.

Exames laboratoriais realizados em maio de 2004 mostraram TSH $<0,03 \mathrm{mUI} / \mathrm{L} ; \mathrm{T} 4$ livre $=4,68 \mathrm{ng} / \mathrm{dl} \mathrm{e}$ T3= 108ng/dl. Ecografia de tireóide com doppler evidenciava tireóide com volume $=20 \mathrm{~cm}^{3}$ e distribuição normal da vasculatura no interior da glândula.

Pela dificuldade de distinção clínica entre as duas formas de tireoidite induzida por amiodarona através dos exames apresentados pelo paciente e, considerando a gravidade do caso e a urgência de tratamento adequado, a dose de metimazol foi mantida em $40 \mathrm{mg} /$ dia e associado prednisona $40 \mathrm{mg} /$ dia.

Após o início da terapia com glicocorticóides, o paciente evoluiu com melhora da sintomatologia clínica e ganho de peso ( $2 \mathrm{~kg}$ em 2 meses). Exames realizados em julho de 2004, 60 dias após a primeira avaliação, mostravam TSH $<0,03 \mathrm{mUI} / \mathrm{L} ;$ T4 livre $=5,64 \mathrm{ng} / \mathrm{dl} \mathrm{e}$ $\mathrm{T} 3=264 \mathrm{ng} / \mathrm{dl}$. Neste momento, o paciente foi mantido com metimazol $40 \mathrm{mg} /$ dia, prednisona $40 \mathrm{mg} /$ dia e associado o carbonato de lítio $300 \mathrm{mg}$ de $12 / 12$ horas.

Em setembro de 2004, 30 dias após o início da terapia com lítio, o paciente retornou clinicamente eutireoidiano, com importante ganho de peso, em torno de $7 \mathrm{~kg}$ em 30 dias, e edema de membros inferiores $(++/ 4)$. Exames laboratoriais demonstravam $\mathrm{TSH}<0,03 \mathrm{mUI} / \mathrm{L} ; \mathrm{T} 4$ livre= $1,7 \mathrm{lng} / \mathrm{dl} ; \mathrm{T} 3=$ $81,05 \mathrm{ng} / \mathrm{dl}$. Por solicitação do serviço de cardiologia, o carbonato de lítio foi suspenso, com redução da dose de prednisona para $20 \mathrm{mg} /$ dia e metimazol para $20 \mathrm{mg} /$ dia. 
Em novembro de 2004, 11 meses após o diagnóstico, mantinha-se clinicamente eutireoidiano, compensado da parte cardiológica. Estava em uso de metimazol $20 \mathrm{mg} /$ dia (iniciado há 11 meses) e prednisona $20 \mathrm{mg} /$ dia (iniciado há 6 meses). Exames laboratoriais realizados, apresentavam $\mathrm{TSH}=0,24 \mathrm{mUI} / \mathrm{L}, \mathrm{T} 4$ livre $=0,73 \mathrm{ng} / \mathrm{dl} ; \mathrm{T} 3=60,2 \mathrm{ng} / \mathrm{dl}$. O paciente foi orientado a suspender o metimazol, com redução gradual da prednisona em esquema de retirada.

Em fevereiro de 2005, 3 meses após o término da terapia com droga antitireoidiana e corticoesteróides e 14 meses após a suspensão da amiodarona, o paciente encontrava-se sem queixas, mantendo-se clínica e laboratorialmente eutireoidiano, com os seguintes exames: $\mathrm{TSH}=3,31 \mathrm{mUI} / \mathrm{L} ; \mathrm{T} 4$ livre $=0,92 \mathrm{ng} / \mathrm{dl}$ e $\mathrm{T} 3=10 \mathrm{lng} / \mathrm{dl}$.

A evolução clínica e laboratorial está resumida na tabela 1 .

\section{DISCUSSĀO}

A amiodarona foi inicialmente utilizada como antianginoso e, posteriormente, como antiarrítmico (l). É amplamente utilizada no tratamento de arritmias ventriculares, taquicardia paroxística supraventricular, fibrilação e flutter atrial. Possui uma meia-vida longa e apresenta, em sua composição, grande quantidade de iodo, sendo que cada molécula contém $37,2 \%$ de iodo (9). O tratamento com amiodarona libera de 7 a $20 \mathrm{mg} /$ dia de iodo, uma concentração 50 a 100 vezes maior que a recomendada (9). A presença de iodo é responsável, juntamente com a homologia estrutural da droga com os hormônios da tireóide, por seus efeitos na função dessa glândula $(4,10,11)$. A amiodarona também atua bloqueando a conversão periférica de T4 para T3 (11).

O hipotireoidismo induzido pela amiodarona ocorre em até $20 \%$ dos pacientes, principalmente naqueles com doença auto-imune de tireóide subjacente $(12,13)$.

A tireotoxicose induzida por amiodarona é mais prevalente em áreas iodo-deficientes, atingindo até $23 \%$ dos pacientes (12). Classifica-se como tipo 1 quando o iodo presente na droga induz a síntese excessiva dos hormônios tireoidianos. Neste caso, a autoimunidade tireoidiana pré-existente é comum e os exames de imagem sugerem normo ou hipervascularização na ecografia com doppler (14) e cintilografia com captação de iodo 131 normal ou aumentada em 24 horas (12). Como terapia, recomenda-se o uso de drogas antitireoidianas (DAT) em altas doses, perclorato de potássio, radiodo ou, nos casos mais graves, tireoidectomia $(2,5,10,12,15,16)$. A ação das DATs tem um efeito tardio em função da grande quantidade de hormônio pré-formado (17). O perclorato de potássio bloqueia competitivamente a entrada de iodo na glândula, porém sem atuar no processo de iodinização. Seu uso foi associado a efeitos colaterais graves, como a anemia aplástica (17). O emprego de radiodo é considerado uma terapia definitiva para a tireotoxicose induzida por amiodarona tipo 1 (17). Recentemente, estudos demonstraram que a associação de TSH recombinante humano (RHTSH) em baixa dose e radiodoterapia ambulatorial é uma forma eficaz e segura de tratamento destes pacientes (18).

Tabela 1. Evolução clínica e laboratorial do paciente.

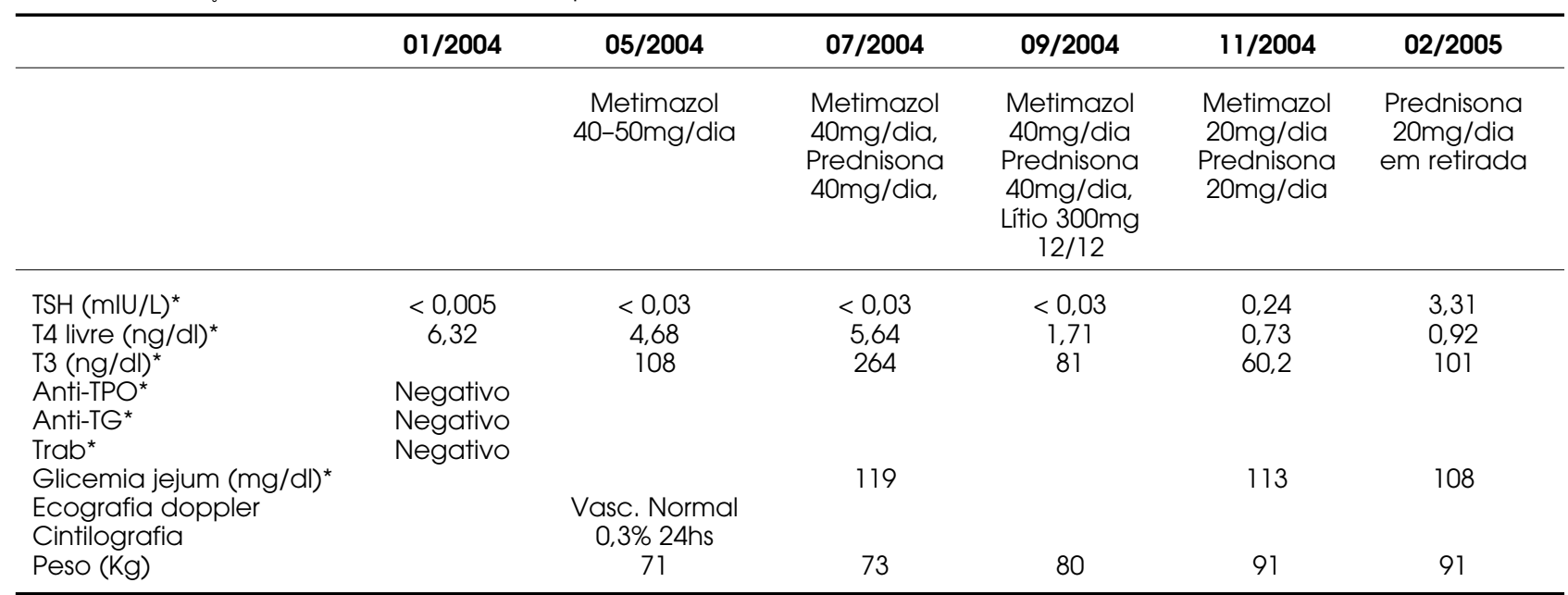

* Valores de referência: TSH= 0,4-4mUl/L; T4 livre= 0,8-1,9ng/dl; T3 total= 70-170ng/dl; Anti-TPO (anti-peroxidase) < 35 Ul/ml; Anti-TG (anti-tireoglobulina)< $40 \mathrm{Ul} / \mathrm{ml}$; Trab< 10\%; glicemia jejum= 74-106mg/dl. 
A tireotoxicose induzida pela tireoidite destrutiva é classificada como tipo 2 . Nestes casos, a tireotoxicose decorre da liberação excessiva de hormônio tireoidiano pré-formado, por um efeito tóxico direto da amiodarona sobre a glândula. $\mathrm{O}$ exame de cintilografia, em geral, demonstra baixa captação de iodo 131 em 24 horas, e a ecografia com doppler apresenta redução na vascularização da glândula (14). A dosagem sérica de interleucina- 6 foi, inicialmente, demonstrada como elevada nos pacientes com tireoidite induzida por amiodarona tipo 2 (19), porém estudo posteriores não reproduziram os mesmos resultados. A tireotoxicose tipo 2 é freqüentemente descrita como uma doença auto-limitada (17). O tratamento consiste no emprego de corticoesteróides em altas doses (17).

A distinção clínica entre as duas formas de tireotoxicose muitas vezes é difícil e, em alguns casos, os pacientes apresentam uma forma mista de doença $(12,17)$. Os casos considerados mistos ou inconclusivos geralmente são tratados com uma combinação de medicamentos, incluindo drogas antitireoidanas, corticoesteróides e, eventualmente, perclorato de potássio (17).

A tireotoxicose é a disfunção tireoidiana induzida pela amiodarona mais grave e associada a maior morbi-mortalidade $(7,17,20)$. As manifestações típicas de taquicardia e fibrilação atrial são especialmente deletérias em pacientes previamente cardiopatas. Em decorrência da elevada meia-vida da droga, a suspensão da amiodarona não normaliza imediatamente a função tireoidiana e, em geral, os pacientes são resistentes ao tratamento habitual, sendo necessário o emprego de terapia combinada com drogas antitireoidianas e corticoesteróides em altas doses $(5,7,12,15$, 16,20). Nos casos refratários, sugere-se a realização de tireoidectomia subtotal $(16,21)$.

O carbonato de lítio é um agente estabilizador do humor, amplamente utilizado para tratamento dos transtornos afetivos bipolares e os episódios de mania ou hipomania. Os efeitos antitireoidianos do lítio envolvem diversos mecanismos: inibição da captação de iodo pela tireóide, inibição da síntese e liberação dos hormônios tireoidianos (8), promoção de auto-imunidade tireoidiana $(8,11,22)$, ativação da desiodase tireoidana (23), modulação da ação dos linfócitos T (22) e inibição da conversão periférica de T4 para T3 (24).

A longo prazo, aproximadamente $50 \%$ dos pacientes tratados com lítio apresentam bócio, 20\% evoluem com hipotireoidismo subclínico e $20 \%$ com hipotireoidismo clínico (25). A incidência de hipotireoidismo é maior nos pacientes com auto-imunidade tireoidiana pré-existente (25).
Atualmente, devido aos potenciais efeitos colaterais, o lítio é proposto como um tratamento de segunda linha para controle da tireotoxicose $(7,26)$.

Dickstein e cols., em 1997, propuseram a terapia com carbonato de lítio associado a droga antitireoidiana para tratamento de pacientes com tireoidite induzida por amiodarona, clinicamente tóxicos (7). Nestes pacientes, a dose inicial de carbonato de lítio foi de $300 \mathrm{mg} \mathrm{3x/dia,} \mathrm{atingindo} \mathrm{até} 1.350 \mathrm{mg} /$ dia. A terapia foi mantida por 4 a 6 semanas após o estabelecimento de eutireoidismo clínico e laboratorial (tempo médio= 10 semanas). Os autores observaram uma significativa redução no tempo de restabelecimento do eutireoidismo nos pacientes tratados com a terapia combinada, quando comparados com os pacientes tratados apenas com droga antitireoidiana. Desta forma, o uso de lítio foi proposto como uma terapia adjuvante segura e eficiente para tratamento dos casos mais graves de tireotoxicose induzida por amiodarona (7).

Relatamos o caso de um paciente, submetido a transplante cardíaco, que desenvolveu um quadro de tireotoxicose induzida por amiodarona. Pela dificuldade no diagnóstico diferencial entre tipo 1 ou tipo 2 e considerando a gravidade do caso, foi instituído como terapia inicial o uso combinado de droga antitireoidiana e corticoesteróides, em altas doses. Em decorrência da ausência de resposta clínica e laboratorial, foi associado o carbonato de lítio. Neste caso, a evolução para eutireoidismo foi rápida e definitiva, compatível com os resultados apresentados por Dickstein e cols. (7), porém com o emprego de carbonato de lítio em baixas doses $(600 \mathrm{mg} / \mathrm{dia})$ e por curto período de tempo (4 semanas).

Concluímos que o carbonato de lítio parece ser uma terapia adjuvante eficaz no controle da tireotoxicose induzida por amiodarona do tipo 1, mesmo em baixas doses e por curto período de tempo, o que pode reduzir a incidência de efeitos colaterais associados ao tratamento.

\section{REFERÊNCIAS}

1. Rosenbaum MB, Chiale PA, Halpern MS, Nau GJ, Przybylski J, Levi RJ, et al. Clinical efficacy of amiodarone as an antiarrhythmic agent. Am J Cardiol 1976;38:934-44.

2. Dickstein G, Amikam S, Riss E, Barzilai D. Thyrotoxicosis induced by amiodarone, a new efficient antiarrhythmic drug with high iodine content. Am J Med Sci 1984;288:14-7.

3. Wilson JS, Podrid PJ. Side effects from amiodarone. Arn Heart J 1991;121:158-71. 
4. Harjai KJ, Licata AA. Effects of amiodarone on thyroid function. Ann Intern Med 1997; 126:63-73.

5. Althaus B, Bucher $\mathrm{H}$, Schon $\mathrm{H}$, Vogt T. Therapy resistance of amiodarone-induced thyrotoxicosis. Schweiz Med Wochenschr 1988; 118:1176-81.

6. Bartalena L, Bogazi F, Martino E. Amiodarone-induced thyrotoxicosis: a difficult diagnostic and therapeutic challenge. Clin Endocrinol 2002;56:23-4.

7. Dickstein G, Shechner C, Adawi F, Kaplan J, Baron E, IshShalom S. Lithium treatment in amiodarone-induced thyrotoxicosis. Am J Med 1997; 102(5):454-8.

8. Johnston AM, Eagles JM. Lithium-associated clinical hypothyroidism: prevalence and risk factors. British J Psych 1999;175:336-9.

9. Rao RH, McReady VR, Spathis G. lodine kinetic studies during amiodarone treatment. J Clin Endocrinol Metab 1986;62:563-7.

10. Trip MD, Wiersinga WM, Plomp TA. Incidence, predictability, and pathogenesis of amiodarone-induced thyrotoxicosis and hypothyroidism. Am J Med 1991;53-7.

11. Bogazzi F, Bartalena L, Gasperi M, Braveman LE, Martino E. The various effects of amiodarone on thyroid function. Thyroid 2001;11:51 1-9.

12. Pearce EN, Farwell AP, Braveman LE. Thyroiditis. N Engl J Med 2003;348:2646-55.

13. Martino E, Aghini-Lombardi F, Mariotti S, Bartalena L, Lenziardi $M$, Ceccarelli $C$, et al. Amiodarone induced hypothyroidism: risk factors and follow-up in 28 cases. Clin Endocrinol 1987;26:227-37.

14. Eaton SE, Euinton HA, Newman CM, Weeteman AP, Bennet WM. Clinical experience of amiodarone-induced thyrotoxicosis over a 3-year period: role of colour-flow Doppler sonography. Clin Endocrinol 2002;56:33-8.

15. Newnham HH, Topliss DJ, Le Grand BA, Chosich N, Harper RW, Stockigt JR. Amiodarone induced hyperthyroidism: assessment of the predictive value of biochemical testing and response to combined therapy using propylthiouracil and potassium perchlorate. Aust NZ J Med 1988; 18:37-44

16. Farwell AP, Abend SL, Huang SKS, Patwardhan NA, Braverman LE. Thyroidectomy for amiodarone-induced thyrotoxicosis. JAMA 1990;263:1526-8.

17. Rajeswaran C, Shelton RJ, Gilbey SG. Management of amiodarone-induced thyrotoxicosis. Swiss Med Wkly 2003:133:579-85.
18. Albino C, Gavioli A. O uso do RHTSH associado a radiodoterapia no tratamento de tireotoxicose induzida por amiodarona (Tipo 1). XI Encontro Brasileiro de Tireóide - apresentação oral. Arq Bras Endocrinol Metab 2004;3(supl. 1):238.

19. Bartalena L, Grasso L, Brogioni S, Aghini-Lombardi F, Braverman LE, Martino E. Serum interleukin-6 in amiodarone-induced thyrotoxicosis. J Clin Endocrinol Metab 1994; 78:423-7.

20. Peche R, Abramowicz M, Unger J. Failure to respond to dexamethasone with fatal consequences, after initial response to multidrug treatment in a case of amiodarone-induced thyrotoxicosis. Am J Med 1993;93:702-3.

21. Claxton S, Sinha SN, Donovan S, Greenway TM, Hoffman $L$, Loughhead $M$, et al. Refractory amiodarone-associated thyrotoxicosis: an indication for thyroidectomy. Aust NZ J Surg 2000;70(3):174-8.

22. Calabrese JR, Gulledge AD, Hahn K, Skwerer R, Kotz M, Schumacher OP, et al. Autoimmune thyroiditis in manicdepressive patients treated with lithium. Am J Psych 1985; 142:1318-21.

23. Frankenfeld TG, Correa da Costa VM, Nascimento-Saba CC, Ortiga-Carvalho TM, Santos RM, Lisboa PC, et al. Thyroid and pituitary thyroxine-5'-deiodinase activity and thyrotrophin secretion in lithium-treated rats. J Endocrinol 2002;174:331-4.

24. Terao T, Oga T, Nozaki S, Onta A, Otsubo Y, Yamamoto $\mathrm{S}$, et al. Possible inhibitory effect of lithium on peripheral conversion of thyroxine to triiodothyronine: a prospective study. Int Clin Psychopharmacol 1995; 10(2):130-5.

25. Bocchetta A, Mossa P, Velluzzi F, Mariotti S, Zompo MD, Loviselli A. A ten-year follow-up of thyroid function in lithium patients. J Clin Psychopharmacol 2001;21:594-8.

26. Lazarus JH, Addison GM, Richards AR, Owen GM. Treatment of thyrotoxicosis with lithium carbonate. Lancet $1974: 2: 1160-2$

\section{Endereço para correspondência:}

Gisah Amaral de Carvalho

Rua Padre Camargo 262

80060-240 Curitiba, PR

E-mail: carvalho@mais.sul.com.br 